\title{
Occupant Workstation Level Energy-use Prediction in Commercial Buildings: Developing and Assessing a New Method to Enable Targeted Energy Efficiency Programs
}

\author{
Ardalan Khosrowpour ${ }^{1}$, Rimas Gulbinas ${ }^{2}$, John E. Taylor ${ }^{*}$
}

${ }^{1}$ Charles E via Department of Civil and Environmental Engineering, Virginia Tech, Blacksburg, VA 24061, United States

${ }^{2}$ The Joan and Irwin Jacobs Technion-Cornell Innovation Institute, Cornell University, New York, NY 10011, United States

*Corresponding Author. Email address: jet@vt.edu.

\begin{abstract}
As buildings become more energy efficient and automated, the role of occupants becomes more significant to assist buildings in reaching their full energy efficiency potential. Predicting occupants' consumption behavior has been identified as one of the most challenging processes in energy efficiency programs and building management system operations. Thus, more accurate behavior learning algorithms for commercial buildings are needed to improve energy efficiency. In this paper, we propose a method to predict occupants' energy-use behavior based on individual energy consumption profiles and assess its potential to increase the effectiveness of energy efficiency programs. The proposed method implements a support vector machine in order to model and predict occupants' short term energy-use patterns and test hypotheses for the existence of a correlation between occupants' entropy, efficiency and prediction accuracy. The results show an average accuracy of $83 \%$ for individual energy-use pattern prediction while being positively correlated with individuals' energy-use behavior. The main contributions of this paper are: 1) proposing and validating a new method to predict individuals' energy-use patterns based on their individual workstation-level energy consumption patterns, and 2) assessing the feasibility and potential of implementing this method to enhance the efficacy of energy efficiency programs to further induce energy efficiency.
\end{abstract}

Keywords: Commercial Buildings; Energy Efficiency; Machine Learning; Occupant Behavior; Support Vector Machine.

(C) 2016. This manuscript version is made available under the Elsevier user license

http://www.elsevier.com/open-access/userlicense/1.0/ 


\section{Introduction}

The commercial building sector is responsible for a substantial portion of energy consumption in the United States. Recent research indicates that, despite technology improvements and tightened standards for this sector, the energy demand continues to grow at an annual rate of $0.8 \%$; this is the fastest growth rate after the industrial sector [1]. The advent of advanced metering technology provides the possibility of building energy monitoring and feedback at various granularities. The most recent generation of wireless sensors facilitate the process of real-time plug load monitoring and feedback. Benefiting from the rich data acquired through these sensors, a thorough analysis on occupants' energy consumption [2,3], energy prediction [4,5], and eventually a more accurate bottom-up building energy prediction could be conducted [6]. This not only could increase energy managers' information about occupants' energy-use, but would also enable them to provide more accurate feedback to occupants and increase the energy efficiency of the building. Building occupant-level energy prediction could significantly improve energy efficiency (EE) programs by providing targeted, personalized, and timely information regarding occupants' behavior [7-13]. This information can considerably increase the effectiveness of such programs while providing a more pleasant user experience for EE programs (e.g. through eco-feedback systems). However, due to the high degree of uncertainty in predicting an individual's energy consumption $[14,15]$, researchers suggest grouping these individuals to improve the prediction accuracy $[16,17]$. Yet, this recommendation is in conflict with the potential for advanced sensors to provide high precision and high resolution data (at the individual occupant level) on consumption in order to empower ecofeedback programs through targeting occupants, and enhance building management system control predictive models.

In this paper, we approach the occupant energy prediction challenge from a behavioral perspective. We propose a method to predict individuals' energy consumption behaviors at a workstation-level. We accomplish this by creating energy-use codebooks based on individuals' historical energy-use, assessing the feasibility of designing a targeted EE program, and introducing a new approach to maximize the effectiveness of EE programs in commercial buildings. Lastly, to validate our method we tested two hypotheses: 1) there exists an interdependency between energy-use pattern prediction accuracy and occupants' energy-use entropy, and 2) there exists an interdependency between energy-use pattern prediction and occupants' energy efficiency. The results of these tests are critical to assess the efficacy of our proposed method to target inefficient occupants in commercial buildings. 


\section{Related Work}

\subsection{Sensor-based Building Energy Monitoring}

Sensor-based energy prediction has gained popularity in both the commercial and residential sector with recent advancements in sensor-based monitoring. However, the body of research is more focused on less granular scales (e.g. building, zone, city, etc.) [16-21]. High resolution sensors facilitate the implementation of bottom-up approaches which can potentially increase the prediction accuracy and provide diagnostics for identifying the areas of inefficiency in buildings [22]. Earlier datasets were captured at a relatively low resolution and building-level granularity, and energy prediction models were only capable of predicting building energy consumption at a high granularity [14]. Later, the metering technology was improved to a higher resolution by monitoring energy-use of central energy consumers in buildings such as HVAC systems, lighting systems, and domestic hot water systems [23-27]. Recent advancements in metering technology have provided an opportunity to meter end-user energy consumption at an appliance-level resolution. Despite these efforts on sensor-based energy feedback, there is still much research to be done to achieve metering infrastructure's full potential to induce energy savings.

\subsection{Building Energy-use Prediction}

In addition to sensor-based feedback approaches to understand energy use, various artificial intelligence (AI) approaches, such as machine learning algorithms, have been implemented to predict short and long term energy consumption of end-users in buildings. Early research in the commercial sector centered on ASHRAE's prediction competition in 1994. That year, MacKay [28] won the energy prediction competition by applying a Bayesian non-linear regression and an automatic relevance determination algorithm. The main focus later shifted toward Neural Networks (NN) and SVM-based algorithms, which represent more robust modeling approaches. Numerous researchers established novel modifications of NN models for energy consumption prediction. Adaptive artificial neural network algorithms [29] and feed forward neural networks [30] are variations that have already been implemented in the literature for energy prediction. However, algorithms are not necessarily the only impacting factor in occupants' energy prediction process. There is a substantial body of knowledge investigating the effects of various features impacting the training and testing of NNs. For example, Wong et al. [23] offered an energy prediction model by including building envelope type as a new input variable to train a model using artificial neural networks. The main differences in NN-based studies are in the input variables, architecture, parameter optimization, etc. In the residential sector, several similar studies on energy forecasting have been conducted. However, according to Edwards et al. [31] who examined various machine learning algorithms to predict residential electricity consumption, the different load shapes between residential and commercial buildings prevent the same algorithm from being effective in both sectors. 
Furthermore, Li et al. [32] compared the strength of SVM and NN algorithms in hourly energy prediction of cooling systems and found SVM as a more accurate algorithm in energy prediction compared to NNs. Moreover, Dong et al. [33] reported the advantages of SVM compared to $\mathrm{NN}$ as smaller sample pool, structural risk minimization approach, and also the fewer number of parameters required to be optimized in training process. Therefore, researchers such as Fan et al. [19] proposed a data mining approach to develop the next-day energy consumption and peak power demand prediction ensemble algorithms. Ensemble algorithms benefit from a combination of ML algorithms to reinforce the learning process and combine multiple underlying basis models, including: multiple linear regression (MLR), auto-regressive integrated moving average (ARIMA), support vector regression (SVR), random forests (RF), multi-layer perceptron (MLP), boosting tree (BT), multivariate adaptive regression splines (MARS), and k-nearest neighbors (KNN). In this work, the ensemble algorithm prediction accuracy outperformed the individual algorithms. There are other advanced methods used to predict occupants' energy consumption in the residential sector such as [34, 35]. Variations of Markov processes (e.g. Hidden Markov Model, Random Markov field, Markov chain, etc.) have been used to develop more comprehensive prediction models with capability of considering prior, transitional, and emission probabilities in models. Among the aforementioned prediction methods, depending on the level of available information, type of data, and the length of data each method can outperform the others. Nevertheless, in this study due to incorporation of energy-use profiles, short length of study, and simplicity of SVM methods we decide to implement a support vector machine method for the sake of prediction.

\subsection{Utilizing Occupant-level Monitoring to Improve and Target Energy Efficiency}

There are a few studies that take advantage of high resolution end-user energy monitoring in residential and commercial buildings with a focus on energy analysis and energy efficiency. Chen et al. [36] utilized an appliance level real-time ecofeedback system to reduce energy consumption and analyze appliances energy-use in residential buildings. In a commercial building setting, Murtagh et al. [37] conducted an eco-feedback study with more than 80 participants, using historical comparison techniques to motivate occupants to save energy. Gulbinas et al. $[2,38]$ investigated the effect of real-time ecofeedback systems coupled with various psychological techniques on occupants' energy consumption behavior in a commercial building. This study examined individuals' energy consumption at a workstation level and provided novel metrics and insights to quantitatively evaluate occupants' energy efficiency. Likewise, Bradley et al [39] studied the effect of ecofeedback systems on individuals' energy consumption in an office environment. At a higher monitoring resolution, Yun et al. [40] designed an appliance-level eco-feedback system (e.g. main desktop computers and monitors, servers, modems, routers, refrigerators, etc.) to improve the occupants' energy awareness and efficiency through various psychological approaches. Moreover, Coleman et al. [41] implemented an appliance-level personalized eco-feedback system to promote 
energy efficient behavior in commercial buildings. These aforementioned studies provide high resolution and personalized feedback such that each occupant can observe the energy-use information related to their own appliances and workstations. In some cases, this includes normative comparison approaches that allow monitoring peers' energy consumption as well. However, there are a limited number of studies that approached promoting energy efficiency in a targeted way to follow a "one size does not fit all" notion as endorsed by numerous researchers [7-13]. In other words, there is a gap of knowledge in that previous studies did not take into account occupants' individual energy-use behavior to provide well-timed, personalized, and targeted energy efficiency feedback to induce further energy efficiency.

Building on this knowledge of existing high resolution and real-time eco-feedback systems $[2,38]$, we developed an energyuse behavior prediction algorithm which is trained based on individuals' historical energy-use codebooks and predicts upcoming time interval energy-use behaviors at an individual-level. The ability to predict energy-use behavior can impact the design of targeted and sustainable feedback systems by providing useful information in a timely manner on potential inefficient energy consumption behaviors and transform the efficacy of EE programs in the near future through promoting curtailment behaviors and inducing higher levels of energy efficiency. For example, such a feedback system could learn and predict when each individual will leave the office and send timely and targeted notification reminders to users that are unlikely to turn off workstation-level equipment. In this study, we utilize a novel occupant classification method to group individuals by their energy-use patterns. Energy-use data is segmented by workday and non-workday hours and energy-use codebooks are created for the morning (i.e. non-working hours), work hours, and evening (i.e. non-working hours). Based on individually classified codebooks, separate models are trained using a support vector machine (SVM) algorithm to infer the future energyuse behavior of segmented clusters of occupants. The main purpose of developing such an algorithm is to leverage the abstracted energy-use profile of each individual and predict the future energy consumption patterns. These patterns, along with the prediction accuracy of our method, provide the information required to evaluate hypotheses designed to validate the proposed methodology. The hypotheses are as follows: 1) an interdependency exists between energy-use pattern prediction accuracy and occupants' behavior, and 2) an interdependency exist between energy-use pattern prediction and occupants' energy efficiency, are tested.

\section{Methodology}

A three step process was taken place as illustrated in Fig. 1. Data collection includes the energy consumption data captured through plug load monitors. The collected energy consumption data was classified for each individual using a 3-step process as described in section 3.2.2. Finally, a support vector machine algorithm used to train and predict individuals' energy load patterns. 


\section{Data Collection}

\section{Classification}
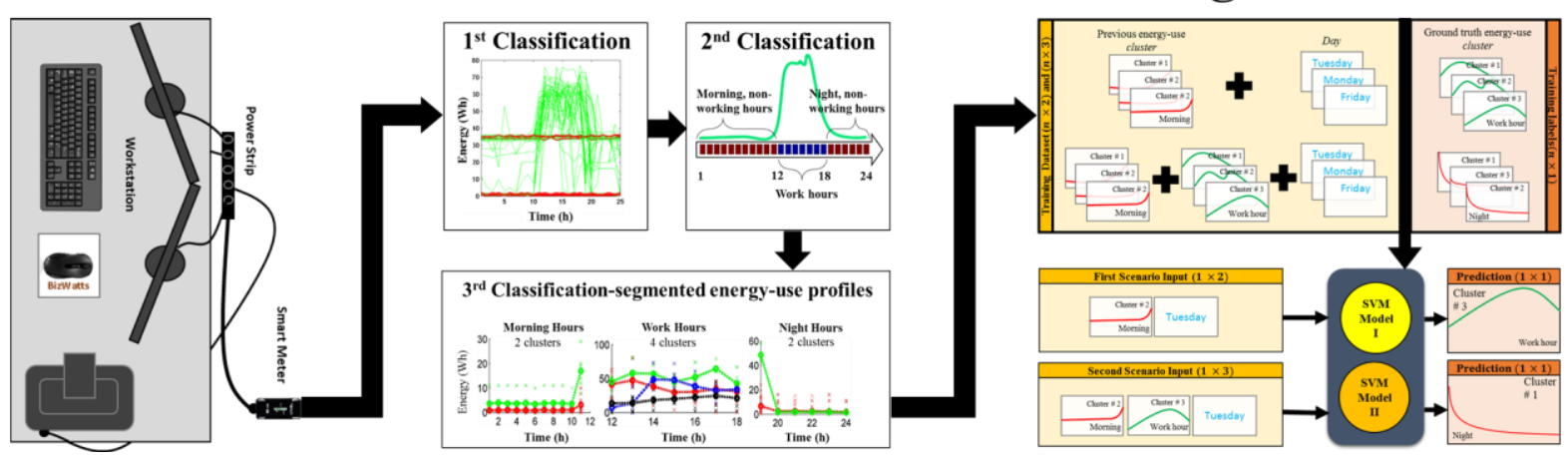

Fig 1. Overview of the process

\subsection{Data Collection}

The data collection was conducted in a 6 story commercial building located in Denver, CO with approximately 115 full-time employees working in 27 non-profit organizations in that building. The workstation appliances were not identical, however, desktop computers or laptops, desk lamps, and in some cases printers were most common appliances. All appliances were connected to a power strip as illustrated in Fig 1 and the power strip energy data was captured through commercially available wireless plug load monitoring sensors. Each group of plug load monitors depending on the location communicate via 900 MHz RF signal with a central router that uploads the data to our database through Ethernet connection. Please refer to [42] for further information regarding infrastructure of our experiment.

\subsection{Classification and Prediction}

The proposed method consists of three key elements described in the following sub-sections. First, occupant energy-use behavior is classified and individual codebooks (i.e. individual energy-use profiles) are generated. Next, a support vector machine is used to learn the occupants' energy-use behavior using a set of features. Finally, energy-use predictions at various resolutions are tested and compared. A main goal of our algorithm is to leverage the existing occupants' energy consumption profiles created through incorporation of multiple modified K-means clustering algorithm on individuals' energy consumption by [2] to predict future energy consumption of each individual. The overview of the algorithms used in [2] are provided in the following subsections. We can then evaluate hypotheses that validate the potential of the approach to enhance energy efficiency programs through predictive models. 


\subsubsection{Daily Load Classification}

Occupants generally follow specific energy-use patterns based on various factors (e.g. working hours, duties, appliances, energy efficiency, etc.). Thus, an individual's energy-use behavior is a variation of a specific set of energy consumption load shapes which slightly vary on a daily basis. The energy load shapes are highly dependent on individuals' energy consumption pattern, work hours, and also the equipment used at each workstation. These repetitive patterns enable codebooks (i.e. energyuse profiles) to be created for each occupant that capture the variability of the entire set of an occupant's load shapes over time [2]. This method could help reduce uncertainty in the predictability of occupants' complex behavior [43, 44]. A $K$-means clustering method is implemented for the means of classification due to application simplicity and the level of accuracy required in this study to distinguish the general pattern of occupants energy consumption. The $K$-means clustering algorithm is to classify $N$ points in $M$ dimensions into $K$ clusters conditioned such that the sum of squares of data point distances to the assigned cluster is minimized. The algorithm requires the dataset and a set of $K$ initial random cluster centers to start. The procedure is to search for $K$-partitions with locally optimal Euclidean distances by moving points from one cluster to another [45]. In our research, the $K$-means clustering algorithm will classify the data points into categories based on hourly energyuse values captured from workstations over each day. The appliances connected to the power strip at each workstation are not identical, and are usually consisted of a desktop computer or laptop, desk lamp, and in some cases a printer. Each classification follows an iterative process combined with a set of thresholds to check the closeness of the cluster centers and determine the $K$ value. The range of $K$ starts from 10 and can go down to 1 based on each iteration's result and the closeness of the clusters. These thresholds will prevent the disaggregation of similar patterns into separate clusters for an individual [2]. The authors refer readers to [2] for further details on the clustering algorithm implemented in this paper. Fig. 2 depicts (a) a sample codebook representing the most dominant energy consumption patterns collected from (b) a non-shared workstation through the duration of the study (i.e. approximately three months) for an individual employee.

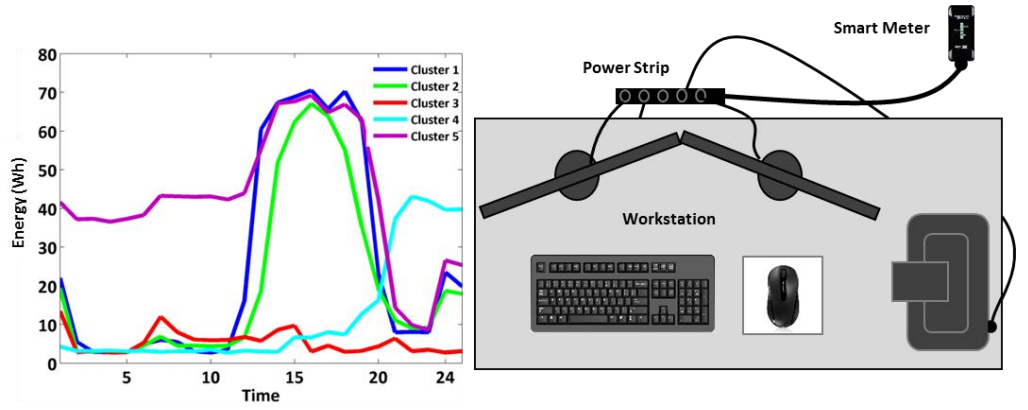

(a)

(b)

Fig 2. (a) Represents employee \#49's energy-use codebook, and (b) illustrates a typical non-shared workstation 


\subsubsection{Segmented Load Classification}

The classification is performed at various stages and resolutions. First, we classify working and non-working days of the week based on pre-set thresholds which check the shape and absolute value of energy loads during the day [2]. Workdays are defined as the days with a large variation of energy-use [2] and each day's data should pass through the same set of filters. The shape filter assures that there is at least a $15 \%$ increase in normalized load shapes and the value threshold checks the absolute difference between the maximum and minimum energy value in each day which has to be greater than 5 watts based on the minimum energy consumption of appliances connected to the smart meter. The second energy-use filtering layer is to assure the prevention of noisy cluster variations in user codebooks by enforcing a minimum threshold on cluster load values [2] . At the second stage, each working day will be segmented into two categories; work hours and non-work hours. The working hours and non-working hours are disaggregated by a k-mean clustering algorithm using Euclidean distance as a separation measure. Finally, the energy profiles are classified when each day's data is segmented to three categories; morning (i.e. non-working hours, before an occupant starts to work), work hours, and night (i.e. non-work hours, after an occupant leaves the building). Each classification is combined with a set of thresholds as explained in the daily load classification section. The daily segmentation is conducted only on working days, as non-working days are mostly flat-shaped and there are no working hours to be separately classified. Fig. 3 illustrates an overview of the segmented classification.

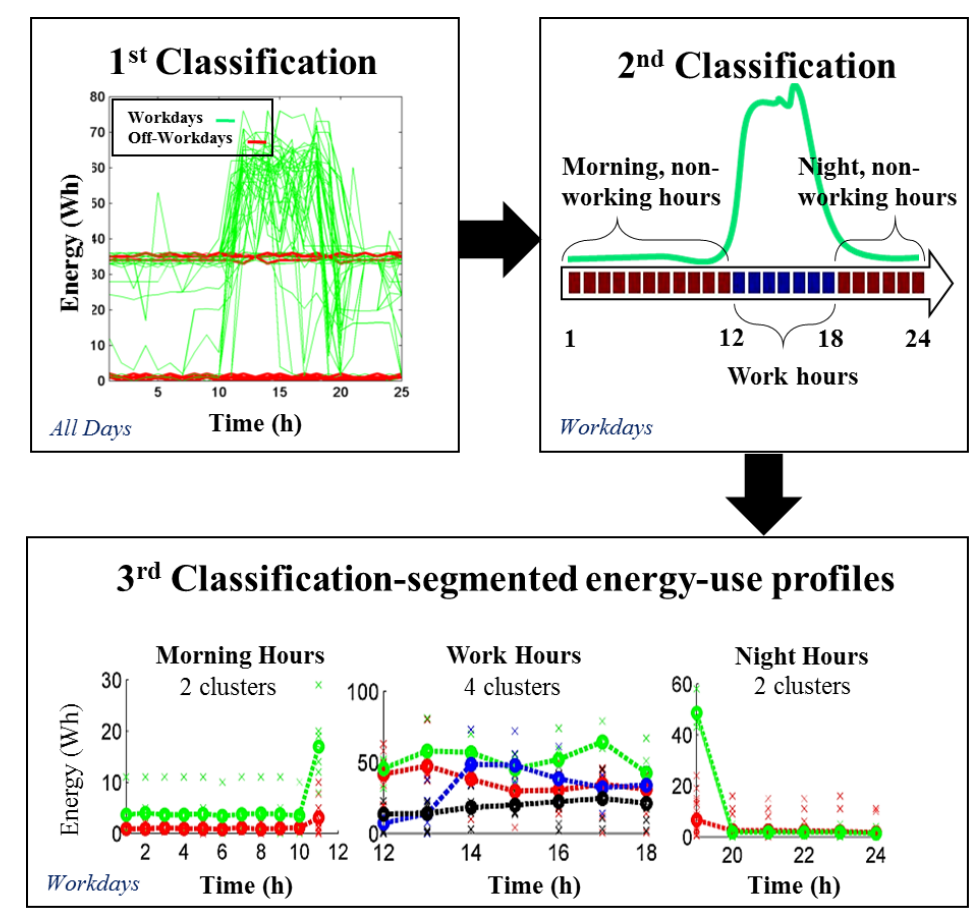

Fig 3. Classification process overview 


\subsubsection{Prediction Algorithm}

As previously mentioned, there are several areas which could potentially benefit from occupant behavior prediction (e.g. tailored EE notification, office appliance diagnosis, more accurate bottom-up energy prediction, etc.). To learn specific models for each occupant, a multi-class one-vs-one Support Vector Machine (SVM) classifier was implemented. A discriminative machine learning algorithm which not only classifies based on the patterns but also optimizes the decision boundaries [46], is necessary to create more accurate models when dealing with inseparable sets of classes. For each occupant $o_{j} \in O$ [where $O$ is the pool of all occupants], we trained a multi-class SVM classifier using given training data $\left\{p_{i}, q_{i}\right\}$. For this classifier, $p_{i} \in P_{j \text {-train }}$, and $P_{j-\text { train }}$ includes all training data points and possible labels are $q_{i}=\{1,2,3, \ldots \mathrm{n}\}$ [where $n$ is the number of energy-use cluster centers (i.e. energy-use patterns) each individual has]. One of the major advantages of SVM is the ability of the algorithm to fit models to the datasets which contain outliers and noise. The concept of slack variables $\xi_{i}$ helps in fitting decision boundary hyperplanes within a soft margin approach. The SVM optimization problem with a linear kernel can be written as:

$$
\begin{gathered}
\min _{w, b} \frac{1}{2}\|w\|^{2}+C \sum_{i=1}^{N} \xi_{i} \\
\text { Subject to: } y_{i}\left(w x_{i}+b\right) \geq 1-\xi_{i} \text { for } i=1, \ldots, N \\
\xi_{i} \geq 0 \text { for } i=1, \ldots, N
\end{gathered}
$$

In (1), $C$ represents a penalty constant which controls the trade-off between the margin size and the training error, and $w$ represents the normal vector to the hyperplane. As a rule of thumb [47], a 5-fold cross validation is used to split our dataset into disjoint training and testing sets with $80 \%$ and $20 \%$ shares, respectively. During the testing phase, we predicted the labels $\{1,2,3, \ldots, \mathrm{n}\}$ for each testing example $p_{i} \in P_{\text {test }}$ with respect to the trained hypothesis for all existing occupants. Implementing a kernel to map the data into a higher dimensional feature space can help to improve the classification performance. Thus, a Gaussian Radial Basis Function (RBF) was chosen over other kernels such as linear, intersection of histograms, polynomial, and chi-square kernels due to a better performance in the prediction. Equation (2) represents the general form of RBF, in addition to the special case of Gaussian kernel.

$$
K\left(x^{(i)}, x^{(j)}\right)=\exp \left(-\gamma\left\|x^{(i)}-x^{(j)}\right\|^{2}\right), \gamma \geq 0, \text { for Gaussian: } \gamma=\frac{1}{2 \sigma^{2}}
$$




\subsection{Daily Energy-Use Prediction}

Individuals follow a certain set of energy-use patterns on a daily basis and each energy-use codebook is different from other occupants. In order to learn energy-use codebooks for each individual, we clustered energy-use patterns for each occupant to create a reduced set of representative daily energy-use load patterns. Furthermore, we took advantage of additional information such as time, working/non-working day, previous day's energy-use pattern, and energy value to create a robust model predicting the next day's energy consumption pattern. A 27 dimensional feature vector (i.e. 25 for previous days energy data points, 1 for previous day, and 1 for previous day's working status), is abstracted to a 3 dimensional space using our classification algorithm [2] and used as an input and mapped through a RBF kernel in order to train the SVM model. The next day's energy-use pattern (i.e. output) is predicted through a 5-fold cross validated process, and the accuracies are calculated. Fig. 4 provides an overview of the process.

(a)

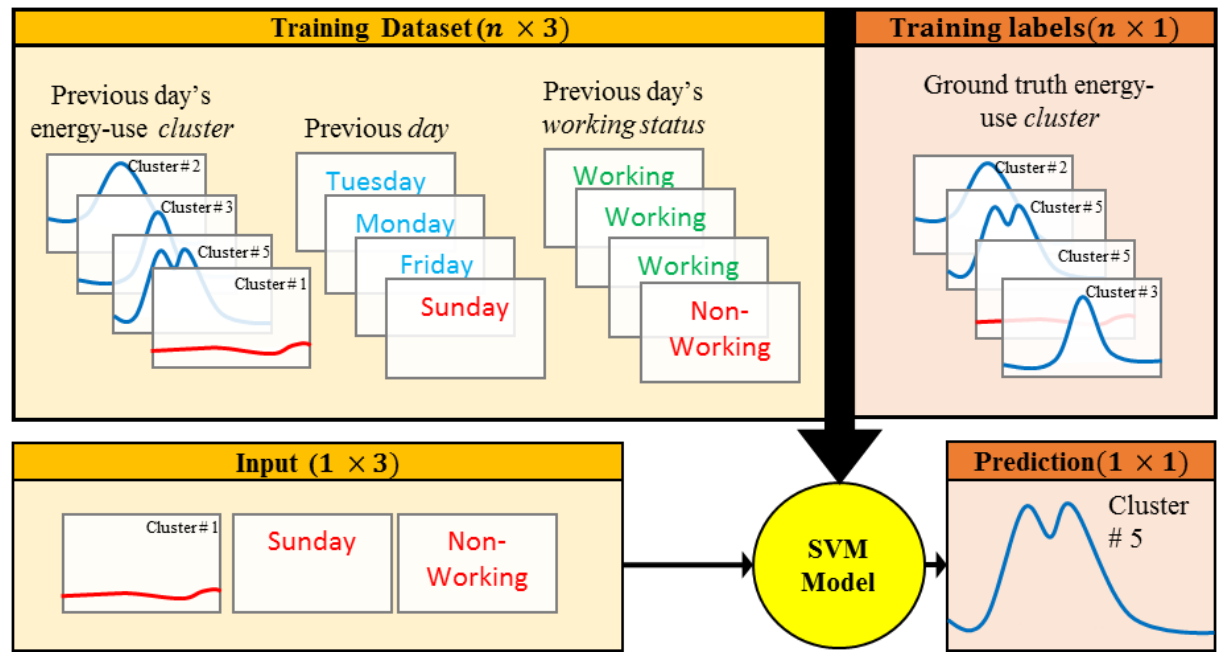

Fig 4. Daily energy-use prediction overview: (a) SVM model training process, and (b) energy-use behavior inference process

\subsection{Segmented Energy-Use Prediction}

The daily profiles were next classified into working vs. non-working hours. There are three segments in each day starting with "morning", before occupants enter the building and start using the appliances at their workstation (i.e. morning nonworking hours). This segment usually starts at 12:00 am and ends in the morning between 8 to $10 \mathrm{am}$. The second part of the day is "working hour" which usually starts between 8 to 10 am and ends around 5 to $7 \mathrm{pm}$. In these hours, occupants are using their appliances and the energy-use level is higher than non-work hours. The last segment, "night" non-work hours, represents the hour range in which occupants leave the building and appliances are usually off or enter power saving mode. All ranges represent approximates of typical schedules and may vary based on the occupants' behavior and working hours. 
This segmentation helps to perform a more precise classification and create more detailed energy-use codebooks. Energy-use data for each occupant was classified for each part of the day as depicted in Fig. 3 and this became part of the input data to train a model based on the occupants' energy-use behavior. We include classification results in the training of specific models for morning and work hours for each occupant (total of two models per occupant) in order to predict the upcoming period's energy-use behavior. The features used to train the SVM models are energy-use clusters and the day of the week. Fig. 5 provides an overview of the algorithm.

(a)

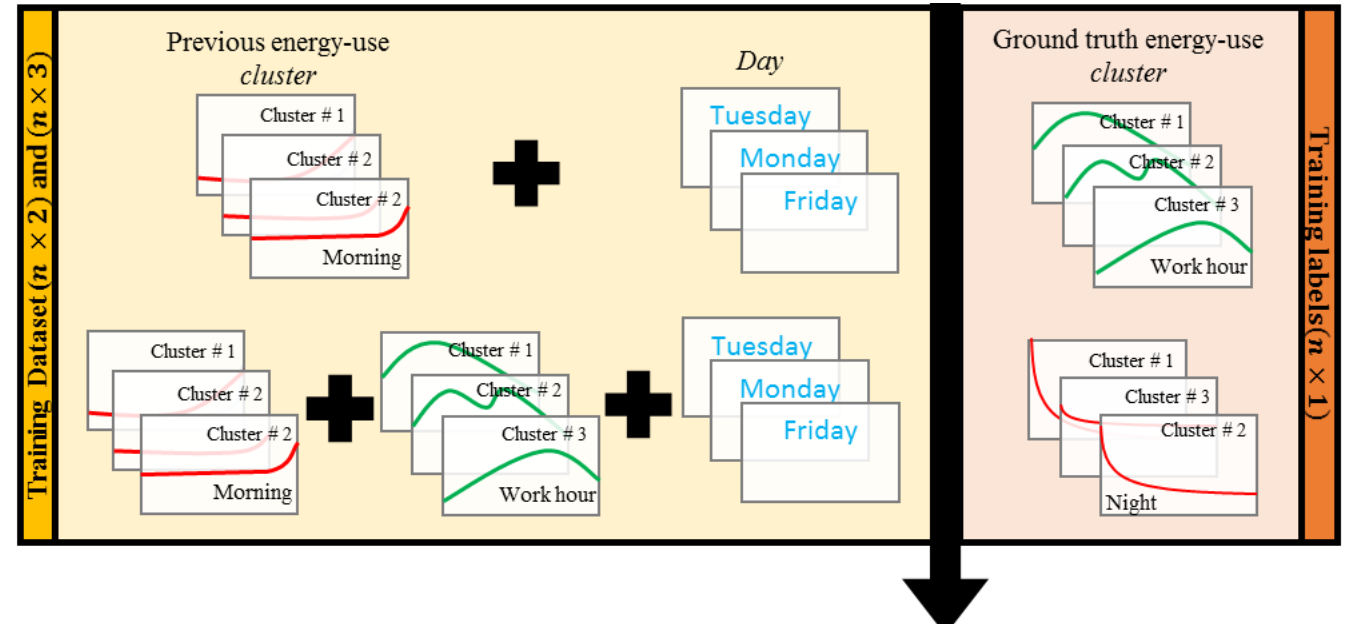

(b)
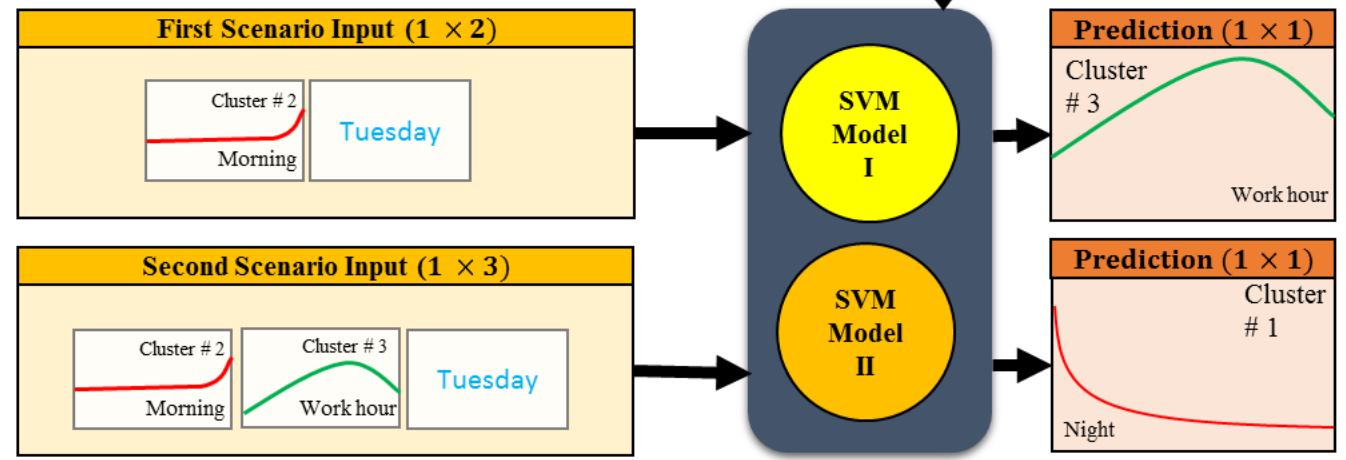

Fig 5. Segmented energy-use prediction overview: (a) SVM model training process, and (b) energy-use behavior inference process

\subsection{Validation}

In order to evaluate the interdependence of occupants' energy-use behavior and the accuracy of energy prediction models and validate our method, we tested two hypotheses: 1) energy-use pattern prediction accuracy and occupants' energy-use entropy are interdependent and 2) energy-use pattern prediction and occupants' energy efficiency are interdependent. The results of these tests are critical to assess the efficacy of our proposed method to target inefficient occupants in commercial buildings. These tests further validate the applicability of such a method to EE programs (e.g. tailored EE notification systems) in order to target the occupants with the highest energy saving potential. The hypotheses are focused on two types of behaviors 
previously defined by Gulbinas et al. [2] as described in section 3.4.3 of this paper-energy efficiency and energy-use entropy.

\subsubsection{Hypotheses}

Hypothesis 1: Occupants' energy-use entropy value is positively correlated with energy-use behavior prediction accuracy.

Hypothesis 2: Occupants' energy efficiency value is positively correlated with energy-use behavior prediction accuracy.

\subsubsection{Hypothesis Testing}

In this section we implement the behavioral measures (i.e. energy efficiency and entropy) based on energy-use patterns of commercial building occupants studied in [2]. Once time across all analyzed days has been effectively

decomposed into various segments (e.g. morning non-work hours, work hours, night non-work hours), and the energy-use for each segment is separated into distinct clusters, as illustrated in Fig. 3, we calculate values representative of a building occupant's energy efficiency and energy-use entropy. Occupants' energy efficiency is defined as the percentage of time each individual spends in the lowest energy consumption cluster. The low energy clusters are defined based on a threshold, in this case is $7 \mathrm{Wh}$ based on an estimate of average power consumed by workstation appliances in off state. The formula used to calculate the level of energy efficiency is provided in [2]. And energy-use entropy is a measure of disorder or uncertainty of energy consumption and a function of the number of times each occupant changes energy-use clusters for each segment of the day over the course of study [2]. The occupants' energy-use entropy, $S_{i}$, is calculated as follows:

$$
\begin{gathered}
s_{l}=-\sum_{i=1}^{k} p\left(C_{i}\right) \log _{10} p\left(C_{i}\right) \\
S_{i}=\frac{\sum_{l=1}^{T} h_{l} s_{l}}{\sum_{l=1}^{T} h_{l}}
\end{gathered}
$$

Where $s_{l}$ is the entropy of segmented range, $l$, that has a length of $h_{l}$ hours and a total of $K$ different behaviors (i.e. clusters) obtained from the clustering algorithm. $T$ is the total number of ranges that entropy is calculated for. Please refer to [2] for further information. Fig. 6 illustrates the mapping of occupants' classification based on energy efficiency and energy-use entropy. 


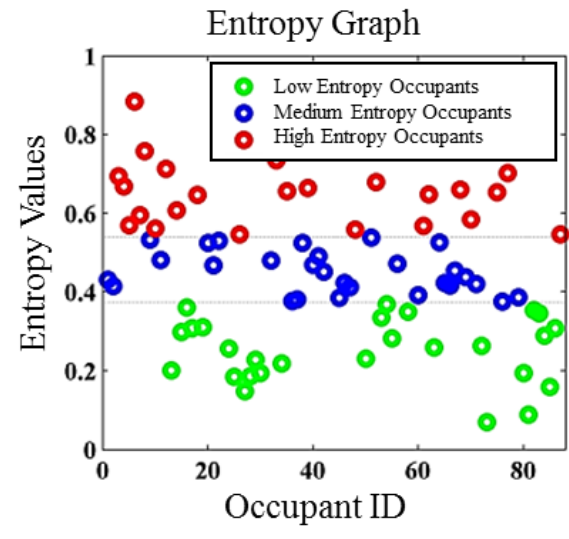

(a)

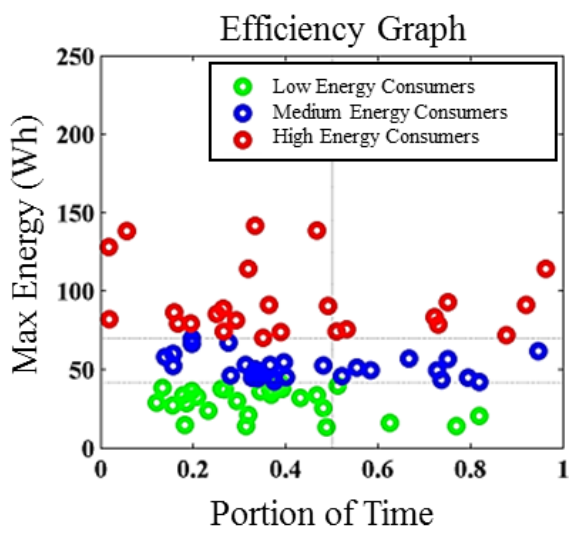

(b)

Fig 6. Occupants' classification based on: (a) energy-use entropy values and (b) energy efficiency

As presented by Fig. 6(a), occupants are separated into three quintiles, low entropy, medium entropy, and high entropy. Fig. 6(b) illustrates the classification of occupants to three quintiles based on the maximum energy consumed in the lowest energy cluster and two categories based on the percentage of time spent in the lowest energy consumption cluster. Based on calculated entropies and energy efficiencies and energy-use behavior prediction as described, we calculate p-values which are the probabilities of getting a correlation as large as the observed values by random chance, when the true correlation is zero. Furthermore, we analyze the prediction accuracies for each group of people to find the best target group that fits a tailored EE notification system. For example, if energy prediction accuracies are low for the group of inefficient occupants, chances are low to engage these individuals in implementing energy efficient behavior through a predictive and targeted notification system.

\section{Results and Discussion}

We utilized LIBSVM [46], an open source machine learning package in order to train and infer energy-use behaviors. Energyuse prediction results were obtained through a 5-fold cross-validation process and the average of all four accuracies will be reported. A confusion matrix and a precision-recall graph were generated for each occupant to evaluate the performance of the model. We refer the reader to [48] for further information on the details of this evaluation method. The predicted energyuse behaviors will be compared to the ground truth data captured by our smart sensors and the coefficient of variation $(\mathrm{CV})$ of the root means square errors, $C V(R M S E) . C V(R M S E)$ is defined as: 


$$
C V(R M S E)=\frac{\sqrt{\frac{1}{N-1} \sum_{i=1}^{N}\left(y_{i}-\hat{y}_{i}\right)^{2}}}{\bar{y}}
$$

where $\hat{y}_{i}$ is the predicted/clustered value, $y_{i}$ is the observed value, $\bar{y}$ is the mean of the observed values and $N$ is the total number of observations.

\subsection{Classification Results}

Initially, clustered energy-use patterns were mapped to the associated cluster centers in order to evaluate the accuracy of the clustering process. The set of cluster centers represent a simplified set of representative profiles. There is a cost associated with simplification and that is an increase in the coefficient of variation. In other words, the clustering process facilitates the task of occupants' energy-use behavior prediction; however, predicted energy-use values could vary slightly from the real values. The average $C V$ calculated based on the cluster centers and ground truth data is 74.07 with a lowest value of 9.60. This is not the best obtainable result based on our classification method, since we did not optimize the $C V$ error-prediction accuracy trade-off; however, when plotting the $C V$ values of each occupant on the $\mathrm{Y}$-axis, and plotting the number of cluster centers for each occupant on the other axis, it is observed that more cluster centers results in lower $C V$ values. Therefore, it could be interpreted that occupants' consistency with specific energy consumption patterns affects the energy-use pattern prediction accuracy and also the energy-use value prediction error.

\subsection{Daily Prediction}

A SVM model was trained for individual occupants based on all the working and non-working days' energy-use. Each model was trained based on the automatically labeled clusters (i.e. energy-use patterns) and evaluated based on a disjoint set of data to prevent overfitting of the models. The overall accuracy of daily energy-use behavior prediction was $81.70 \%$ with the highest prediction rate of $100 \%$. In order to demonstrate the trend between the prediction accuracy and number of clusters for each occupant, a box plot is graphed in Fig. 7. These results show a decrease in the energy-use prediction accuracy as the number of cluster centers increases. Therefore, there is a trade-off between the energy-use behavior prediction and our classification $C V$. The higher the number of cluster centers there are for each occupant, the lower the $C V$ is. On the other hand, the higher the number of cluster centers there are, the lower the prediction accuracy is. We therefore seek to determine the optimum points at which our system reaches its maximum performance by bounding the number of cluster centers [49]. This approach effectively captures occupant energy-use behavior, and increases the energy value prediction accuracy of our system. 


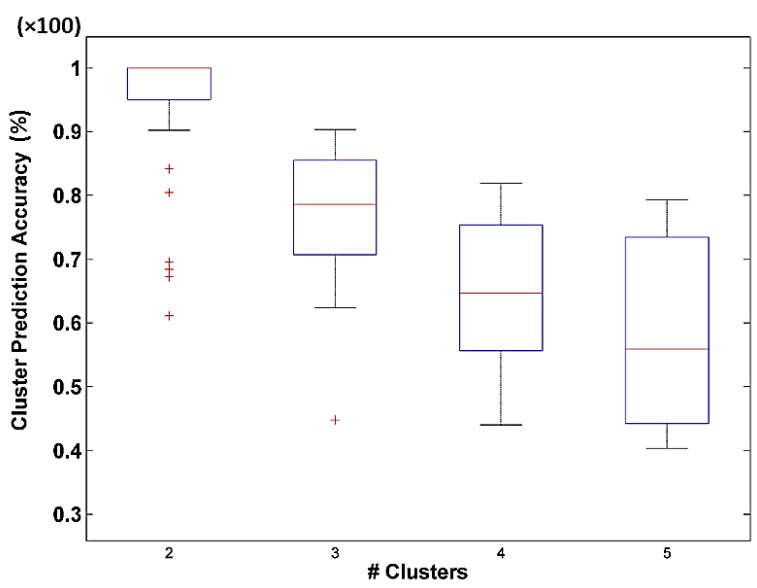

Fig 7.Daily energy-use prediction accuracy vs. number of clusters for each individual

Another measure by which we can evaluate our prediction algorithm is a confusion matrix. Confusion matrices present the false positive detections and the rate of confusion among various patterns for each occupant. This would help us identify the most confused patterns and find the source of confusion to improve the classification and prediction algorithms. Based on our investigation, the misclassifications in our model happen because of similar energy shapes and values in the training dataset, similar repetitions of the same patterns on the same day of the week, and lack of sample data. Fig. 8 illustrates a sample data analysis for occupant \#49 in which the average prediction accuracy is $78.4 \%$. Based on the confusion matrix, the maximum confusion was between clusters number 2 and 1. The energy-use cluster centers are presented in Fig. 8(a). Cluster \#1 and \#2 are very similar in terms of shape and value; however, cluster \#2 has been repeated thirteen times more than cluster \#1 in our dataset and this encouraged our algorithm to choose cluster \#2 more confidently under similar conditions.

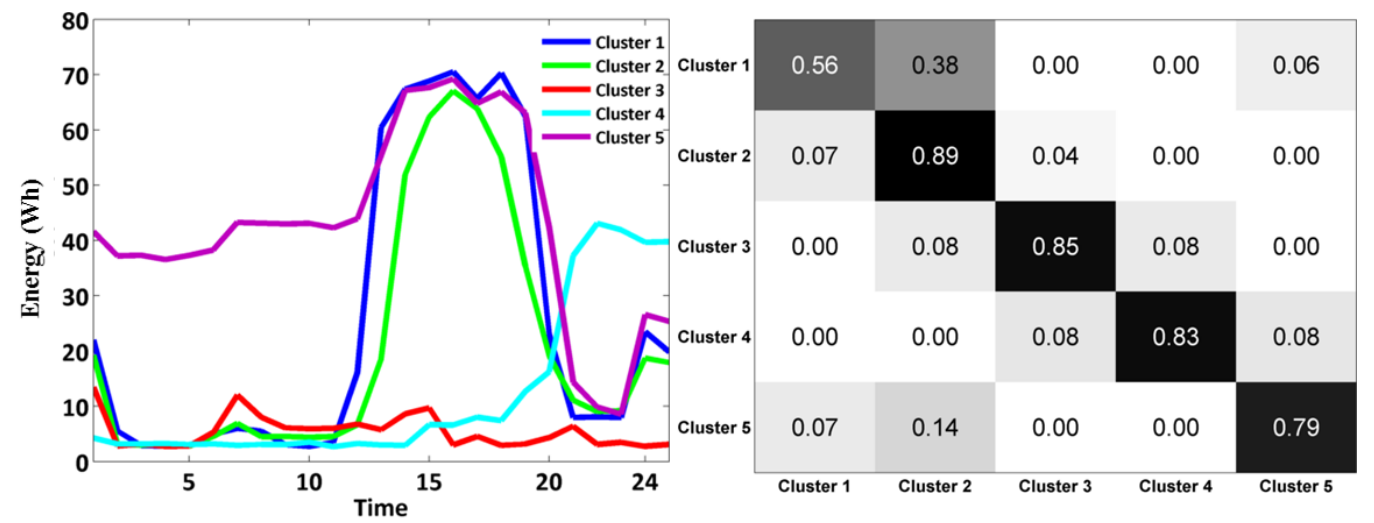

(a)

(b)

Fig 8. (a) Sample energy-use codebook for an occupant \#49; (b) confusion matrix for energy-use prediction for occupant \#49 
A precision-recall curve was also plotted for each occupant to study the overall performance of our algorithm. This was done by dividing the predicted results based on their confidence score given by SVM and considering that each interval has the same number of sample data points. The ideal plot is the one which has a precision close to 1 regardless of the recall value, this ideal condition shows that prediction accuracy is always $100 \%$ at all prediction confidence thresholds. As illustrated in Fig. 9, the precision rate drops as we increase the recall. When the recall rate is low, the decrease in the precision rate is extremely undesirable and shows the weakness of the model. In occupant \#49's case, the precision fluctuates around $80 \%$ for most of the recall rates below $80 \%$. However, when the recall rate passes $80 \%$ the precision drops precipitously.

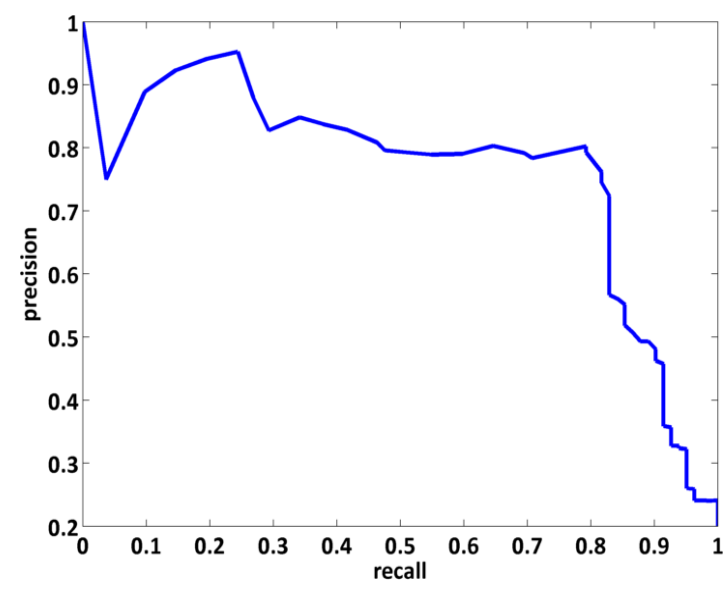

Fig 9. Precision-recall graph for energy-use prediction for occupant \#49

To evaluate the prediction accuracy, we calculated the $C V$ for predicted energy-use clusters by benchmarking the ground truth data. The result shows a lower performance due to the existing inaccuracies in the prediction algorithm with an average $C V$ of 91.15 and the lowest $C V$ of 14.03 . The coefficient of variation is calculated for predicted energy-use clusters by benchmarking the associated ground truth cluster centers. The result shows an average $C V$ of 45.08 and the highest performance of $C V=0$.

\subsection{Segmented Prediction}

After exploring the energy-use behavior prediction and coefficient of variation for daily predictions, we are proposing a higher resolution classification and prediction as described in section 3.3 on Segmented Energy-use Prediction. The prediction algorithm will be applied to each segment of the day, and later the predictions are combined together to present the daily energy-use codebooks. Based on the collected data in the morning, the work hour energy-use pattern is predicted (i.e. first scenario), and based on the work hour energy-use, the night hour energy-use data is predicted (i.e. second scenario). There is 
little need for morning energy prediction (i.e. non-working hours before employees go to work) since it is very unlikely that employees work over night or come back to the office after they leave for the day. This assumes that the morning energy-use pattern should follow the previous night energy-use pattern.

A multi-class one-vs-one support vector classifier was implemented to train mutually exclusive models for the aforementioned scenarios. The first one is to predict the work hour energy-use pattern and the second one to predict the night hour energy-use pattern. 5-fold cross validated results are reported for each prediction based on disjoint training and testing datasets. The average accuracy of the work hour prediction model is $88.01 \%$ with the highest accuracy of $100 \%$ and an average prediction accuracy of $77.99 \%$ for the night hour energy-use prediction with the maximum accuracy of $98.08 \%$. The work hour predictions obtained lower accuracy since there is more intra-class variability in work hour behaviors for each occupant. Occupants' work hours energy-use behavior could vary from 2 to 7 different clusters, while non-work hour clusters typically varied between 2 to 3 .

Energy-use prediction accuracies were plotted versus the number of energy-use clusters for each individual. As graphed in Fig. 10, a decreasing trend of prediction accuracies for both (a) night hours and (b) work hour energy-use prediction can be observed while increasing the number of clusters. Therefore, the lower the number of clusters, the more accurate our prediction algorithm would work.
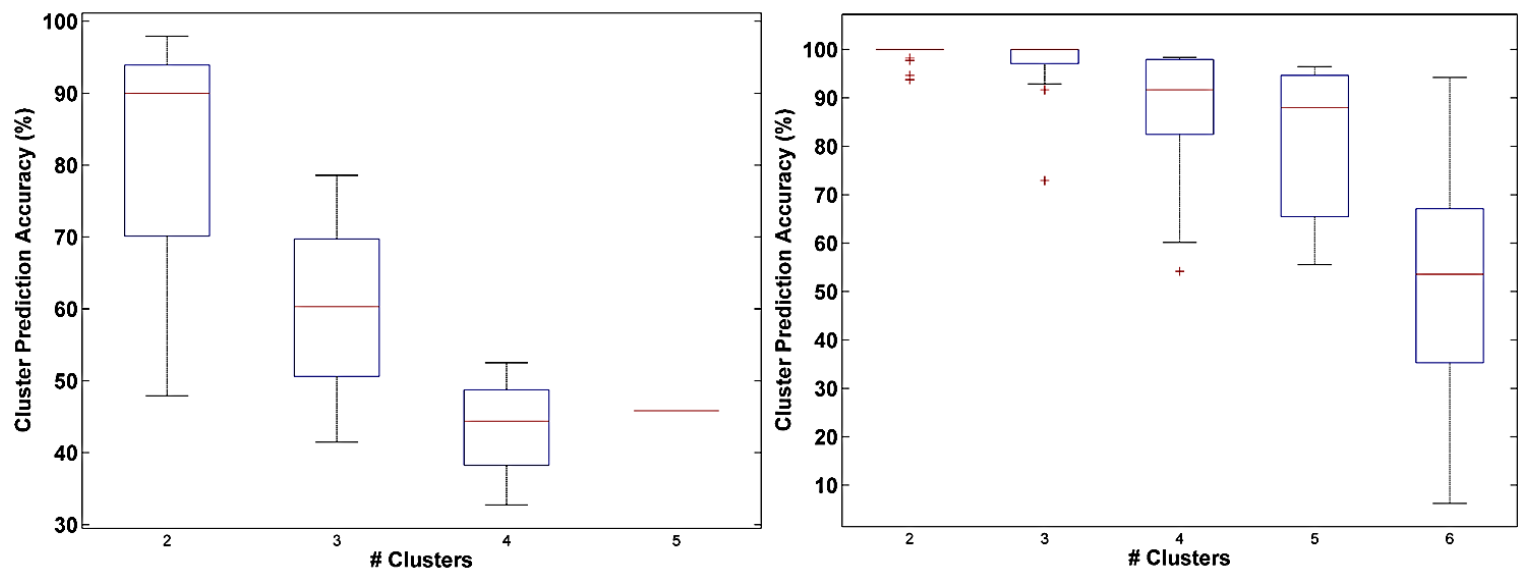

Fig 10. Prediction accuracy of segmented model versus the number of cluster centers for each individual; (a) night hour and (b) work hour

In addition to the prediction accuracy, the coefficient of variation $(\mathrm{CV})$ is calculated for each occupant to observe how differences in the prediction compared to the ground truth data collected by the sensors. Fig. 11 graphs the $39^{\text {th }}$ day energyuse prediction for occupant \#80 superimposed on (a) the associated cluster centers and (b) the ground truth data. In this particular case, work hour and night hour energy-use predictions are both correct. Nevertheless, the prediction varies in terms 
of the value compared to ground truth data. As depicted in Fig. 11, our algorithm predicted that occupant \#80 in all probability will forget to turn off the appliances at his/her workstation. This type of behavior could most likely be detected if an occupant has repeatedly forgotten to turn off the appliances and this behavior could be observed enough times in the dataset to train a model.

The ability to predict occupant energy-use patterns which reflect certain behaviors provides useful information for energy efficiency (EE) programs and automated notification and feedback systems. Three $C V$ values are calculated to measure the goodness of our prediction algorithm in terms of energy values rather than energy-use patterns. As expected, cluster centers and the predicted clusters $C V$ have the lowest value of 34.47 which outperforms the same $C V$ value calculated in section B for the daily resolution prediction. The $C V$ value calculated between cluster centers and the ground truth data (i.e. hourly energy data) was 48.03. Finally, the $C V$ value calculated based on the ground truth data and the predicted cluster centers has the greatest value of 61.45 compared to the previous two. However, all of these $C V$ s outperform the daily resolution $C V$ s as described in previous sections, which indicates a more accurate energy value prediction for the segmented prediction method. Remarkably, the energy-use behavioral prediction accuracy of $83.00 \%$ for segmented load prediction outperforms the prediction accuracy of $81.70 \%$ for the daily load predictions. A better performance by segmented load prediction algorithm suggests the benefit of higher resolution energy monitoring and creating more informative models to enhance EE programs through occupants' behavior prediction and potentially providing more accurate bottom-up energy prediction approaches.

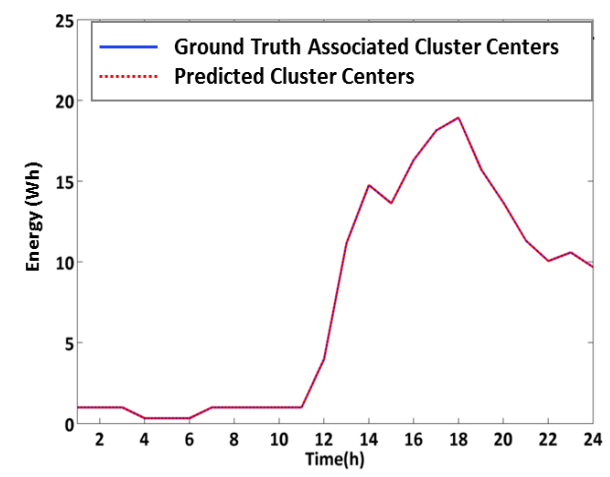

(a)

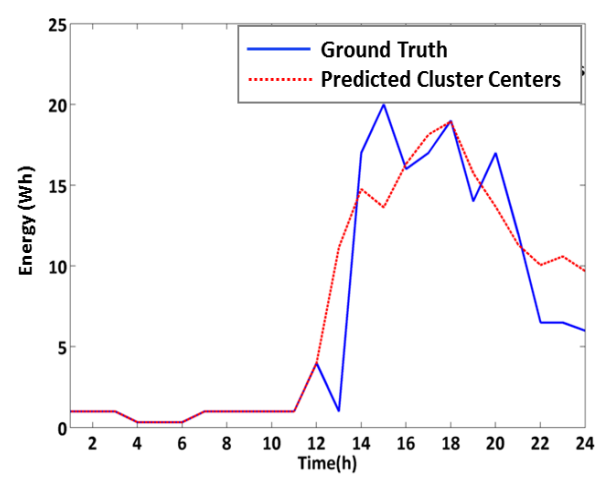

(b)

Fig 11. Joint graph of segmented predictions for the 39th day of occupant \#80

\subsection{Hypotheses Test Results}

In this section we present the results obtained from cross-validated SVM models for 87 occupants for two different scenarios ${ }^{1}$ as presented in Fig. 12. Six different categories of occupant energy-use are modeled and tested separately. As illustrated in

\footnotetext{
${ }^{1}$ First Scenario: using morning hour data as an input and predicting working hour energy-use cluster. Second scenario: implementing work hour data as an input and predicting night hours energy-use cluster.
} 
Fig. 6, these 6 categories consisted of: low entropy occupants, medium entropy occupants, high entropy occupants, low energy consumers, medium energy consumers, and high energy consumers; determined by ranking occupants based on their performances in each category and dividing them into three quantiles. For each occupant a 5-fold cross-validated accuracy was calculated and compared to the rest of the group members. Fig. 12(a) presents the occupants' energy-use entropy which has been linearly scaled from $[0,1]$ to $[100,0]$ for the sake of easier visualization and comparison with SVM prediction accuracies. Thus, we define energy-use predictability, which is a linear transformation of our entropy values, as the consistency at which an occupant follows particular energy-use load profiles. The closer the predictability value is to 100 , the more consistent an occupant's behavior is and vice versa. Equation (6) is the function used to transform the entropy to predictability data. The green bars in Fig. 12(a) represent the most consistent occupants, the blue bars show medially consistent occupants and the red bars are representative of occupants with low consistency in energy-use behavior. Fig. 12(b) demonstrates the prediction accuracies for each individual and is color-coded based on the predictability classification noted above.

$$
\text { Predictability }=100 \times(1-\text { Entropy })
$$

(a)

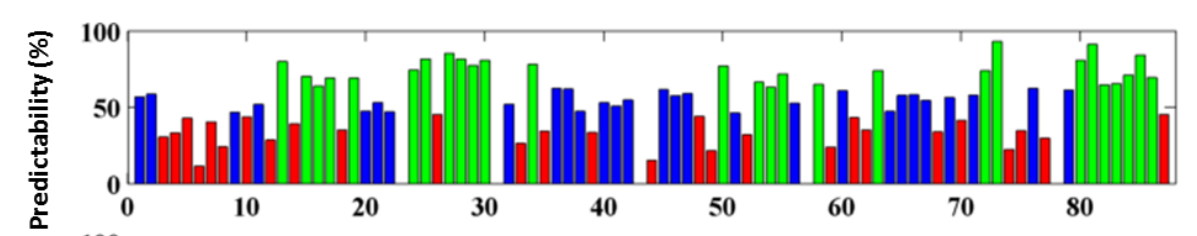

(b)

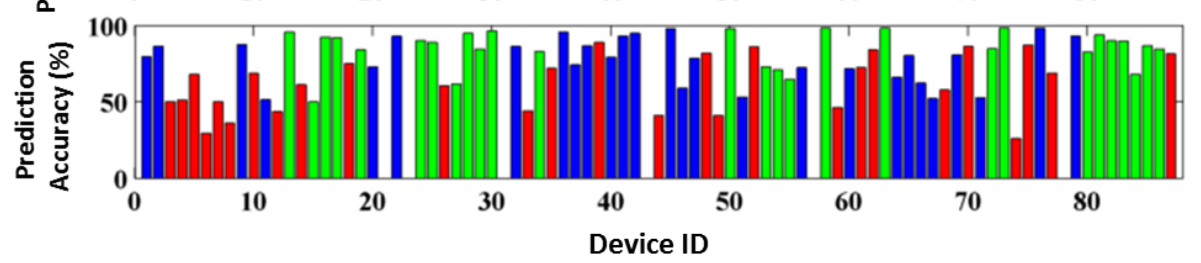

Fig 12. Comparison of: (a) predictability and (b) prediction accuracy.

Fig. 13(a) depicts the prediction accuracy for occupants with high predictability (most consistent energy-use behavior). Fig. 13(b) graphs medium predictable occupants and 13(c) low predictable occupants. The SVM prediction accuracy obtained for various groups of occupants classified based on their energy-use predictability and energy consumption rate are presented in Table 1. Table 1(a) shows the accuracies for prediction of work hour energy, while Table 1(b) represents SVM prediction accuracies for night hour classification. It should be considered that the results presented in Table 1 might be slightly different compared to the previously reported results due to the randomness of the cross validation function in selecting the training and resting datasets. 
Lastly, statistical significance of correlations between behavioral classification and prediction accuracies were calculated. The p-value obtained for energy-use predictability and prediction accuracy correlation is below 0.01 and the p-value calculated for energy efficiency and prediction accuracy turned out to be different for various scenarios. Obtained p-values for energy efficiency correlation ranged from 0.00016 to 0.2257 for different conditions (i.e. scenarios). Therefore, more analysis is required to evaluate a possible positive correlation between energy efficiency of occupants and energy-use behavior prediction accuracy. Moreover, a review of the high, medium, and low energy consumption categories defined based on occupants energy consumption quantiles indicates that the SVM prediction accuracies are in the same range. This indicates that there is strong potential for targeting high energy consumers through automated EE programs through accurate prediction of their energy-use behaviors.

Table 1. SVM prediction accuracy for various occupant classes

(a) Features: Morning hour energy data $\rightarrow$ Prediction: Work hour energy-use cluster

\begin{tabular}{|c|c|c|c|}
\hline Predictability Class & SVM Prediction Accuracy & Energy consumption Class & SVM Prediction Accuracy \\
\hline High Predictability & $88.68 \%$ & High Energy Consumption & $79.95 \%$ \\
\hline Medium Predictability & $78.32 \%$ & Medium Energy Consumption & $72.82 \%$ \\
\hline Low Predictability & $63.79 \%$ & Low Energy Consumption & $78.28 \%$ \\
\hline
\end{tabular}

(b) Features: Work hour energy data $\rightarrow$ Prediction: Night hour energy-use cluster

\begin{tabular}{|c|c|c|c|}
\hline Predictability Class & SVM Prediction Accuracy & Energy consumption Class & SVM Prediction Accuracy \\
\hline High Predictability & $90.02 \%$ & High Energy Consumption & $84.23 \%$ \\
\hline Medium Predictability & $83.02 \%$ & Medium Energy Consumption & $84.41 \%$ \\
\hline Low Predictability & $79.05 \%$ & Low Energy Consumption & $83.41 \%$ \\
\hline
\end{tabular}

(a)

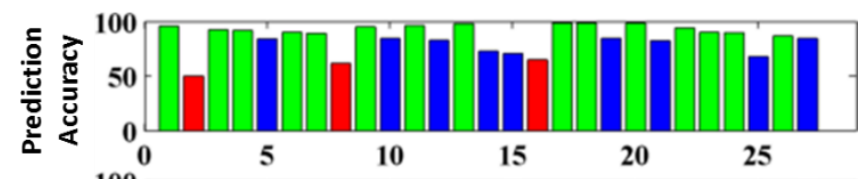

(b)

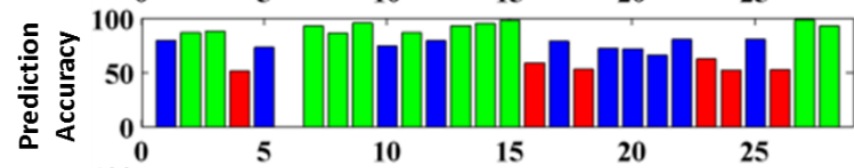

(c)

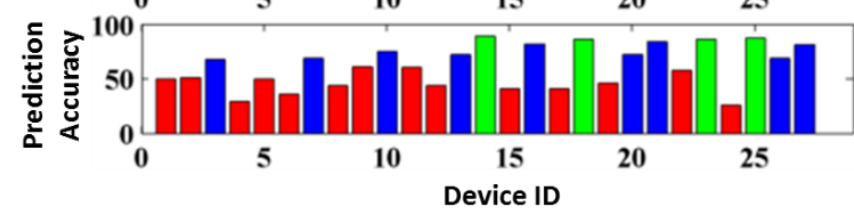

Fig 13. Comparison between: (a) high predictable, (b) medium predictable, and (c) low predictable occupants' SVM prediction accuracy 
Occupants' energy-use behavior predictability is a critical factor in capturing value from an automated EE program. However, it is important to predict inefficient occupants' energy-use behavior as they have a relatively higher potential for energy savings. The best target for such systems are low efficient occupants whose behavior are highly predictable. Fig. 14 maps the occupants' distribution in a $3 \times 3$ matrix based on the predictability and efficiency measures. Nearly $50 \%$ of low efficient occupants are highly predictable and $36.6 \%$ are medially predictable (i.e. occupants fallen into second predictability quantile); the rest fall into the low predictable category. In other words, there is potential to target over $85 \%$ of inefficient occupants through targeted EE programs such as tailored energy efficiency notification systems. Furthermore, over 50\% of medially efficient occupants fall into the high and medium predictability classes which adds further potential conservation targets to the targeted population. Based on our estimate, up to $45 \%$ of the occupants could be targeted effectively with automated EE programs and the rest are either already highly efficient or are not using energy predictably. Therefore, based on the energy efficiency and predictability analysis conducted with respect to occupants' energy-use pattern prediction accuracy, the proposed method has the potential to contribute to the advancement of EE programs by merging an occupant behavior learning algorithm with an individual-level energy consumption feedback system. Moreover, building on such energy-use behavior learning algorithms, a robust occupancy detection system [50] could be developed which significantly improves commercial building energy efficiency through optimization of the building management system operation.

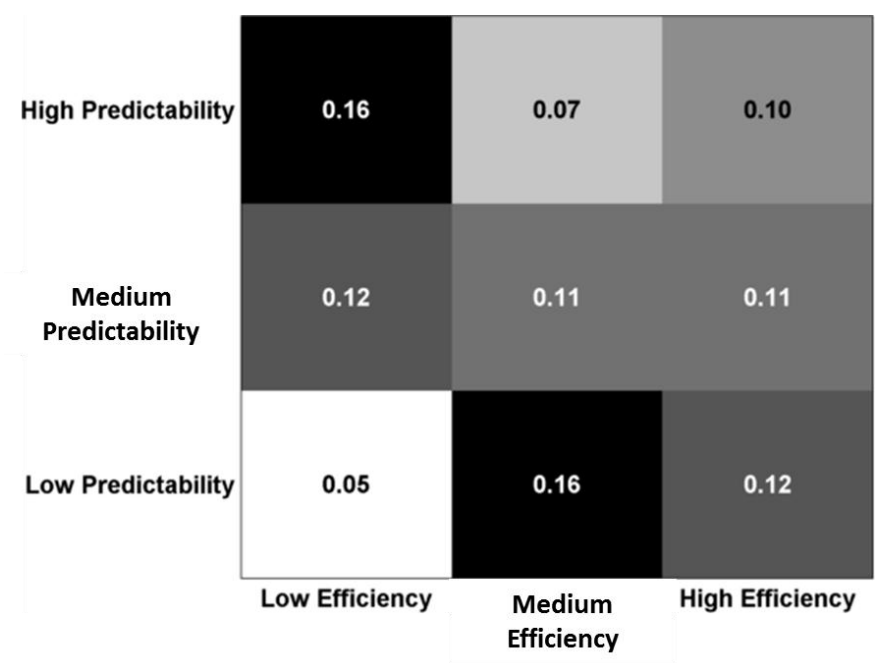

Fig 14. Occupant distribution map based on the energy-use behavior factors

\section{Limitations \& Future Research}

The data collection was limited to assessing occupants' workstation energy efficiency potential. However, in commercial office buildings with decentralized lighting and HVAC systems controlled by occupants, the impact of such EE programs 
can be extended beyond workstations. There are a few limitations which need to be addressed in order to improve the underlying algorithms in our method. It is not our intent to outperform state-of-the-art prediction algorithms. Improving upon prediction accuracy is, however, a planned area of future research. The SVM model may be improved by adding extra information, such as, demographic information of the occupants, occupancy sensor data, weather conditions, mobility patterns, and appliance energy-use disaggregation. Furthermore, there are instances where the system predicts false positives and false negatives. At this stage, we are only predicting atomic energy-use behaviors; however, in the future we could improve our model through implementing a Markov model and enable the prediction of long sequences of energy-use patterns and occupant behavior. This could potentially increase the accuracy of the prediction by creating conditional models based on a wide range of behavioral event probabilities. The focus of this work was not outperforming other energy prediction

algorithms. However, to compare the accuracy of our method in bottom up energy prediction, we would like to compare the $C V$ values obtained from our prediction to other data mining and regression models in future research, including: support vector regression, random forest, multivariate adaptive regression lines, etc. trained based on the current dataset. Future research should seek to improve the current energy-use pattern prediction accuracy to a level where it can be used to implement this automated tailored notification system in experimental case studies to evaluate the result of targeted interventions on near term and sustained energy savings. Potential benefits of a predictive energy feedback system are in providing timely and tailored feedbacks to individuals based on the predicted behaviors to prevent inefficient energy consumption habits such as leaving on the lights, printers, and personal computers while not in the office. Moreover, if such predictive feedback program is combined with an online control system, it can facilitate remote energy savings through web, computer, and cellphone platforms. Another advantage of such EE programs can be found in more comprehensive predictive systems where machine learning algorithms are trained based on individuals' classes and characteristics. This enables a targeted and tailored EE program based on psychological and demographic differences. To illustrate, one might be assigned to a normative comparison treatment, while other may achieve more energy-use reductions if targeted with monetary incentives. Unfortunately, our Institutional Review Board human subject research approval did not allow for collecting information regarding characteristics and demographics of the participants in our study. This may be an interesting avenue for future research.

\section{Conclusion}

Recent advancements in building technology and monitoring systems have benefited building energy efficiency programs. However, considering that people spend more than $90 \%$ of their time indoors [51] coupled with the significant correlation between behavior and energy consumption [43], occupants remain a major factor impacting building energy consumption 
and associated $\mathrm{CO}_{2}$ emissions. Occupant classification and behavioral analysis are new approaches that address the occupant behavioral inefficiency challenge and could potentially empower prediction models to better learn and infer occupants' energy-use behavior. Energy efficiency programs are evolving toward more personalized data collection and analysis with higher resolution data. Therefore, there is a need for increasingly automated energy efficiency programs through integration of high technology hardware, robust software, and fundamentals of behavioral science to handle the massive data streams extracted from buildings and occupants. In this paper, we were inspired from previous studies in the field of customer classification and load profiling [2,52-54] and developed a method that integrated energy-use classification and prediction algorithms to predict individual energy-use behaviors in a commercial building. We found that nearly $50 \%$ of low efficient occupants are highly predictable and another $36.6 \%$ are medially predictable. This high level of individual energy use predictability could result in a new approach to energy efficiency programs by predicting the occupants' behavior and enabling predictive and relevant energy saving notifications. Sustainable behavioral change is a challenge which has not yet been thoroughly addressed, thus limiting the effectiveness of behavior-oriented energy feedback systems $[8,37,39-41,55$,

56]. The presented results in this paper hold promise to provide an automated and sustainable solution to occupant-level energy efficiency programs (e.g. automated tailored energy efficiency notification system) and open a new venue to enable targeted interventions by categorizing the occupants based on their consumption behaviors. Moreover, merging such predictive models with the state-of-the-art occupant energy feedback methods such as online appliance control system [57], energy-use disaggregation [41, 57-59], and cellphone-based feedback [60] can potentially induce higher levels of energy efficiency. Future research should incorporate a more comprehensive version of the behavior prediction algorithm trained on additional occupant data including mobility patterns, comfort preferences, work schedules, demographics, and workstation energy-use to examine whether more substantial and sustainable reductions in energy consumption can be realized through automated and targeted feedback. Occupant behavior learning algorithms not only will enable a cost-effective occupant-based energy saving in commercial buildings, but also will empower the next generation of occupant-centered building management systems which operate more efficient and comfortable intelligent buildings in the future.

\section{Acknowledgments}

This material is based upon work supported by the Department of Energy Building Technologies Program and the National Science Foundation under Grant No. 1142379. Any opinions, findings, and conclusions or recommendations expressed in this material are those of the authors and do not necessarily reflect the views of the Department of Energy or the National 
Science Foundation. The authors would like to thank the Alliance for Sustainable Colorado for hosting the study that provided the building occupant data used in the experimental data analysis in this paper.

\section{References}

1. $\quad$ U.S.EIA, Annual Energy Outlook 2014 Early Release Overview. 2014.

2. Gulbinas, R., A. Khosrowpour, and J.E. Taylor, Segmentation and Classification of Commercial Building Occupants by Energy-Use Efficiency and Predictability. IEEE Transaction on Smart Grids, 2014.

3. Kwac, J., J. Flora, and R. Rajagopal, Household energy consumption segmentation using hourly data. Smart Grid, IEEE Transactions on, 2014. 5(1): p. 420-430.

4. $\quad$ Chen, J. and C. Ahn, Assessing Occupants' Energy Load Variation through Existing Wireless Network Infrastructure in Commercial and Educational Buildings. Energy and Buildings, 2014.

5. Milenkovic, M. and O. Amft, Recognizing energy-related activities using sensors commonly installed in office buildings. Procedia Computer Science, 2013. 19: p. 669-677.

6. Hong, T. Occupant behavior: impact on energy use of private offices. in ASim 2012-1st Asia conference of International Building Performance Simulation Association., Shanghai, China, 11/25/12-11/27/12. 2014.

7. Vassileva, I., et al., The impact of consumers' feedback preferences on domestic electricity consumption. Applied Energy, 2012. 93: p. 575-582.

8. Hargreaves, T., M. Nye, and J. Burgess, Keeping energy visible? Exploring how householders interact with feedback from smart energy monitors in the longer term. Energy Policy, 2013. 52(0): p. 126-134.

9. Quintal, F., et al., WATTSBurning: design and evaluation of an innovative eco-feedback system, in HumanComputer Interaction-INTERACT 2013. 2013, Springer. p. 453-470.

10. He, H.A., S. Greenberg, and E.M. Huang. One size does not fit all: applying the transtheoretical model to energy feedback technology design. in Proceedings of the SIGCHI Conference on Human Factors in Computing Systems. 2010. ACM.

11. van Dam, S.S., C.A. Bakker, and J.D.M. van Hal, Home energy monitors: impact over the medium-term. Building Research \& Information, 2010. 38(5): p. 458-469.

12. Allcott, H. and S. Mullainathan, Behavioral science and energy policy. Science, 2010. 327(5970): p. 1204-1205.

13. Allcott, H., Social norms and energy conservation. Journal of Public Economics, 2011. 95(9-10): p. 1082-1095.

14. Swan, L.G. and V.I. Ugursal, Modeling of end-use energy consumption in the residential sector: A review of modeling techniques. Renewable and Sustainable Energy Reviews, 2009. 13(8): p. 1819-1835.

15. Hoes, P., et al., User behavior in whole building simulation. Energy and Buildings, 2009. 41(3): p. 295-302.

16. Jain, R.K., et al., Forecasting energy consumption of multi-family residential buildings using support vector regression: Investigating the impact of temporal and spatial monitoring granularity on performance accuracy. Applied Energy, 2014. 123(0): p. 168-178.

17. Sevlian, R. and R. Rajagopal, Short Term Electricity Load Forecasting on Varying Levels of Aggregation. arXiv preprint arXiv:1404.0058, 2014.

18. Zhao, H.-X. and F. Magoules. New parallel support vector regression for predicting building energy consumption. in Computational Intelligence in Multicriteria Decision-Making (MDCM), 2011 IEEE Symposium on. 2011. IEEE.

19. Fan, C., F. Xiao, and S. Wang, Development of prediction models for next-day building energy consumption and peak power demand using data mining techniques. Applied Energy, 2014. 127: p. 1-10.

20. Zhao, H.-X. and F. Magoulès, A review on the prediction of building energy consumption. Renewable and Sustainable Energy Reviews, 2012. 16(6): p. 3586-3592.

21. Kavaklioglu, K., Modeling and prediction of Turkey's electricity consumption using Support Vector Regression. Applied Energy, 2011. 88(1): p. 368-375.

22. Lee, W.-Y., J.M. House, and N.-H. Kyong, Subsystem level fault diagnosis of a building's air-handling unit using general regression neural networks. Applied Energy, 2004. 77(2): p. 153-170.

23. Wong, S., K.K. Wan, and T.N. Lam, Artificial neural networks for energy analysis of office buildings with daylighting. Applied Energy, 2010. 87(2): p. 551-557.

24. Li, Q., et al., Applying support vector machine to predict hourly cooling load in the building. Applied Energy, 2009. 86(10): p. 2249-2256.

25. Ben-Nakhi, A.E. and M.A. Mahmoud, Cooling load prediction for buildings using general regression neural networks. Energy Conversion and Management, 2004. 45(13): p. 2127-2141.

26. Aydinalp, M., V. Ismet Ugursal, and A.S. Fung, Modeling of the appliance, lighting, and space-cooling energy consumptions in the residential sector using neural networks. Applied Energy, 2002. 71(2): p. 87-110. 
27. Xuemei, L., et al. Building cooling load forecasting using fuzzy support vector machine and fuzzy c-mean clustering. in Computer and Communication Technologies in Agriculture Engineering (CCTAE), 2010 International Conference On. 2010. IEEE.

28. MacKay, D.J., Bayesian nonlinear modeling for the prediction competition. ASHRAE transactions, 1994. 100(2): p. 1053-1062.

29. Yang, J., H. Rivard, and R. Zmeureanu, On-line building energy prediction using adaptive artificial neural networks. Energy and buildings, 2005. 37(12): p. 1250-1259.

30. Karatasou, S., M. Santamouris, and V. Geros, Modeling and predicting building's energy use with artificial neural networks: methods and results. Energy and Buildings, 2006. 38(8): p. 949-958.

31. Edwards, R.E., J. New, and L.E. Parker, Predicting future hourly residential electrical consumption: A machine learning case study. Energy and Buildings, 2012. 49: p. 591-603.

32. Li, Q., et al., Predicting hourly cooling load in the building: a comparison of support vector machine and different artificial neural networks. Energy Conversion and Management, 2009. 50(1): p. 90-96.

33. Dong, B., C. Cao, and S.E. Lee, Applying support vector machines to predict building energy consumption in tropical region. Energy and Buildings, 2005. 37(5): p. 545-553.

34. Albert, A. and R. Rajagopal, Smart meter driven segmentation: What your consumption says about you. Power Systems, IEEE Transactions on, 2013. 28(4): p. 4019-4030.

35. Tanimoto, J., A. Hagishima, and H. Sagara, A methodology for peak energy requirement considering actual variation of occupants' behavior schedules. Building and Environment, 2008. 43(4): p. 610-619.

36. Chen, V.L., M.A. Delmas, and W.J. Kaiser, Real-time, appliance-level electricity use feedback system: How to engage users? Energy and Buildings, 2014. 70: p. 455-462.

37. Murtagh, N., et al., Individual energy use and feedback in an office setting: A field trial. Energy Policy, 2013. 62 : p. 717-728.

38. Gulbinas, R., R.K. Jain, and J.E. Taylor, "BizWatts": A modular socio-technical energy management system for empowering commercial building occupants to conserve energy. Applied Energy, 2014.

39. Bradley, P., M. Leach, and S. Fudge, The Role of Social Norms in Incentivising Energy Reduction in Organizations. 2014.

40. Yun, R., et al., Toward the design of a dashboard to promote environmentally sustainable behavior among office workers, in Persuasive Technology. 2013, Springer. p. 246-252.

41. Coleman, M.J., et al., Promoting behaviour change through personalized energy feedback in offices. Building Research \& Information, 2013. 41(6): p. 637-651.

42. Gulbinas, R., R. Jain, and J. Taylor, BizWatts: A modular socio-technical energy management system for empowering commercial building occupants to conserve energy. Applied Energy, 2014. 136: p. 1076-1084.

43. Virote, J. and R. Neves-Silva, Stochastic models for building energy prediction based on occupant behavior assessment. Energy and Buildings, 2012. 53: p. 183-193.

44. Mahdavi, A. and C. Pröglhöf, User behaviour and energy performance in buildings. Wien, Austria: Internationalen Energiewirtschaftstagung an der TU Wien (IEWT), 2009.

45. Hartigan, J.A. and M.A. Wong, Algorithm AS 136: A k-means clustering algorithm. Applied statistics, 1979: p. 100-108.

46. Chang, C.-C. and C.-J. Lin, LIBSVM: a library for support vector machines. ACM Transactions on Intelligent Systems and Technology (TIST), 2011. 2(3): p. 27.

47. Murphy, K.P., Machine learning: a probabilistic perspective. 2012: MIT press.

48. Manning, C.D., P. Raghavan, and H. Schütze, Introduction to information retrieval. Vol. 1. 2008: Cambridge university press Cambridge.

49. Khosrowpour, A., R. Gulbinas, and J.E. Taylor. An Empirical Comparison of Internal and External Load Profile Codebook Coverage of Building Occupant Energy-Use Behavior. in Computing in Civil Engineering 2015. 2015. ASCE.

50. Agarwal, Y., et al. Occupancy-driven energy management for smart building automation. in Proceedings of the 2nd ACM Workshop on Embedded Sensing Systems for Energy-Efficiency in Building. 2010. ACM.

51. U.S.EPA The Inside Story: A Guide to Indoor Air Quality. 2012.

52. Albert, A., R. Rajagopal, and R. Sevlian. Segmenting consumers using smart meter data. in Proceedings of the Third ACM Workshop on Embedded Sensing Systems for Energy-Efficiency in Buildings. 2011. ACM.

53. Mutanen, A., et al., Customer classification and load profiling method for distribution systems. Power Delivery, IEEE Transactions on, 2011. 26(3): p. 1755-1763.

54. Quilumba, F.L., et al., Using Smart Meter Data to Improve the Accuracy of Intraday Load Forecasting Considering Customer Behavior Similarities. Smart Grid, IEEE Transactions on, 2015. 6(2): p. 911-918.

55. Gulbinas, R. and J.E. Taylor, Effects of real-time eco-feedback and organizational network dynamics on energy efficient behavior in commercial buildings. Energy and Buildings, 2014. 84(0): p. 493-500. 
56. Allcott, H. and T.T. Rogers, How Long Do Treatment Effects Last? Persistence and Durability of a Descriptive Norms Intervention's Effect on Energy Conservation. 2012.

57. Yun, R., et al. Beyond eco-feedback: adding online manual and automated controls to promote workplace sustainability. in Proceedings of the 33rd Annual ACM Conference on Human Factors in Computing Systems. 2015. ACM.

58. Giri, S. and M. Bergés, An energy estimation framework for event-based methods in Non-Intrusive Load Monitoring. Energy Conversion and Management, 2015. 90: p. 488-498.

59. Berges Gonzalez, M.E., A framework for enabling energy-aware facilities through minimally-intrusive approaches. 2010.

60. Petkov, P., et al. Motivating domestic energy conservation through comparative, community-based feedback in mobile and social media. in Proceedings of the 5th International Conference on Communities and Technologies. 2011. ACM. 